DOI: https://doi.org/10.11144/Javeriana.upsy18-4.ieap

\title{
Inteligencia emocional y ajuste psicológico en estudiantes: nivel académico y rama de estudios*
}

\section{Emotional Intelligence and Psychological Adjustment in Students: Academic Level and Field of Study}

Recepción: 28 Junio 2018 | Aceptación: 03 Noviembre 2019

\author{
Mónica Carballeira Abella ${ }^{a}$ \\ Universidad de La Laguna, España \\ ORCID: http://orcid.org/0000-0002-3525-441X \\ Betsabé Marrero Carreira \\ Universidad de La Laguna, España \\ ORCID: http://orcid.org/0000-0003-0659-2951 \\ Desiré Abrante Rodríguez \\ Universidad de La Laguna, España \\ ORCID: http://orcid.org/0000-0001-7579-4034
} a Autor de correspondencia. Correo electrónico:
mabella@ull.edu.es

Para citar este artículo: Carballeira Abella, M., Marrero Carreira, B., \& Abrante Rodríguez, D. (2019). Inteligencia emocional y ajuste psicológico en estudiantes: nivel académico y rama de estudios. Universitas Psychologica, 18(4), 1-14. https:// doi.org /10.11144/Javeriana.upsy18-4.ieap

\section{RESUMEN}

El objetivo principal de este estudio ha sido analizar la inteligencia emocional (IE) de 147 estudiantes adultos de distintos niveles educativos. Se realizaron correlaciones de las tres escalas de IE -percepción, comprensión y regulación emocional- con variables de personalidad, autoestima, apoyo social y sintomatología general. La regulación emocional fue la escala de IE con mayor número de relaciones, fundamentalmente con responsabilidad, apertura a la experiencia, extraversión, autoestima y apoyo social. No se encontraron diferencias en las puntuaciones de IE entre los tres niveles académicos -formación profesional, grado universitario y máster universitario-. El contraste entre estudiantes con puntuaciones extremas en cada una de las tres escalas de IE, mostró patrones diferenciales en características sociodemográficas, autoestima, apoyo social y sintomatología general. Además, se llevó a cabo un contraste en las escalas de IE, autoestima y apoyo social de aquellos participantes de la muestra original de las ramas de Ciencias Sociales y Ciencias de la Salud, frente a una muestra de estudiantes de Ingeniería Informática. Estos mostraron menor apoyo social y percepción emocional. Los presentes hallazgos aportan información complementaria a los estudios realizados al respecto, con el fin de implementar intervenciones psicológicas dirigidas a promover la inteligencia emocional entre estudiantes de diferentes niveles académicos. Palabras clave

inteligencia emocional; personalidad; autoestima; apoyo social; sintomatología.

\section{ABSTRACT}

The main purpose has been to analyze the emotional intelligence -EI- in 147 adult students, from different academic levels: vocational students, undergraduate students and master's degree students. The associations of the three EI scales -emotional attention, emotional clarity and emotional repair- with other variables, such as personality, self-esteem, social 
support and general symptomatology were analyzed. Emotional repair was the EI scale that stablished greater relationships, especially with conscientiousness, openness to experience, extraversion, self-esteem and social support. No differences were found in comparing the EI scores among the three academic levels -vocational students, undergraduate students and master's degree students-. Some differences were found between those students with extreme scores in EI, on sociodemographic characteristics, self-esteem, social support and general symptomatology. Additionally, a contrast study considering the three scales of IE, self-esteem and social support, between a sample of Computer Engineering students and those participants from the original sample studying Social or Health Sciences, showed less social support and emotional attention among Computer Engineering students than Social or Health Sciences degrees. These findings give complementary information to the research carried out in this regard, in order to implement psychological interventions to promote emotional intelligence among students from different academic levels.

Keywords

emotional intelligence; personality; self-esteem; social support; symptomatology.

$\mathrm{Al}$ hacer referencia al concepto de inteligencia, normalmente se tiende a aludir al cociente intelectual (CI) como medida de la estimación numérica de la inteligencia general. Aun así, sigue siendo difícil delimitar este concepto debido a que no solo depende de las habilidades intelectuales expresadas en la medida del CI, sino también de otros factores. Así, durante las últimas décadas, se han explicado dichas diferencias, dando como resultado la consideración de distintos tipos de inteligencia (Molero-Moreno, Saiz-Vicente, \& Esteban-Martínez, 1998). Para tener un buen rendimiento y/o éxito en la vida, además de las habilidades cognitivas incluidas en dicho constructo, también se requieren habilidades como autocontrol, entusiasmo, capacidad para automotivación y perseverancia, englobado todo ello bajo el constructo de Inteligencia Emocional (IE), con un componente social importante que va más allá del CI (Mayer \& Salovey, 1993).

Cuando se habla del concepto de IE, generalmente se está haciendo referencia al trabajo de Goleman (1995), en el que incluyen cinco competencias: autoconocimiento emocional, autocontrol emocional, automotivación, empatía y habilidades sociales (Egido, 2018). Sin embargo, Salovey y Mayer (1993) se consideran los principales precursores de la IE que, a principios de los años 90, parten de los estudios iniciados por Thorndike en los años 20, desarrollando una definición en la que hacían referencia a la inteligencia en un nivel global, teniendo en cuenta tanto componentes cognitivos (pensamiento, memoria y percepción) como emocionales, afectivos, sociales y personales (Extremera-Pacheco \& Fernández-Berrocal, 2003), ambos necesarios para nuestra capacidad de éxito y adaptación a la vida. Ante las distintas divulgaciones surgidas y que alejaban el concepto de IE de su acercamiento teórico y científico, el grupo de Mayer y Salovey reformuló su propia definición: "La capacidad para procesar la información emocional con exactitud y eficacia, incluyendo la capacidad para percibir, asimilar, comprender y regular emociones" (Mayer, Salovey, \& Caruso, 2000, p. 107).

Existe una doble consideración del concepto de IE entre: (i) modelos de habilidades (para percibir, comprender y manejar la información procedente de las emociones) (Mayer, Caruso, \& Salovey, 1999) y (ii) modelos mixtos o basados en rasgos de personalidad (estables, temperamentales, de competencias socio-emocionales, aspectos motivaciones y habilidades cognitivas) (Bar-On, 2000; Boyatziz, Goleman, \& Rhee, 2000; Goleman, 1995).

El Modelo de Inteligencia Emocional de Salovey y Mayer (1997), está constituido por un conjunto de habilidades emocionales formando un continuo, desde la percepción emocional hasta la regulación emocional, pasando por la comprensión emocional. La percepción emocional permite reconocer nuestras emociones \# estados y sensaciones fisiológicas y cognitivas\# e identificar las expresadas por los demás. La comprensión emocional ayuda a interpretar el significado de las emociones complejas etiquetándolas, reconocer el paso de un estado emocional a otro y la aparición de sentimientos simultáneos y contradictorios, así como las 
emociones secundarias, las causas de un estado emocional determinado y saber en qué categoría emocional se sitúan. Finalmente, la regulación emocional, de mayor complejidad, hace lograr crecimiento emocional e intelectual y manejar el mundo interpersonal e intrapersonal, a partir de la regulación de emociones propias y ajenas, tanto positivas como negativas, en función de la información que las acompañan. La regulación emocional (los estados emocionales) permite lograr crecimiento emocional e intelectual, y manejar el mundo interpersonal e intrapersonal, a partir de la regulación de emociones propias y ajenas, tanto positivas como negativas, en función de la información que las acompañan (Goleman \& Davidson, 2017).

La IE es un constructo importante para explicar el ajuste psicosocial de los individuos (Salguero, Fernández-Berrocal, RuizAranda, Castillo, \& Palomera, 2011). En población universitaria, se han hallado relaciones importantes positivas con empatía y negativas con inhibición emocional (FernándezBerrocal, Extremera-Pacheco, \& Ramos, 2004) \#especialmente de comprensión y regulación emocional\#, que desempeñan un papel importante en el bienestar subjetivo de los individuos (Serrano \& Andreu, 2016) y también en salud física (Mikolajczak, Luminet, \& Menil, 2006) o determinados hábitos saludables (Martins, Ramalho, \& Morin, 2010; Schutte, Malouff, Thorsteinsson, Bhullar, \& Rooke, 2007). Indudablemente, la IE es importante en situaciones con implicación emocional (Lenaghan, Buda, \& Eisner, 2007). Así, los trastornos del estado de ánimo y el consumo de sustancias se relacionan negativamente con IE (Lizeretti, Oberst, Chamarro, \& Farriols, 2006), el estrés percibido (Extremera-Pacheco, Durán, \& Rey, 2007) y también la fobia social, los trastornos de pánico y los obsesivo-compulsivos (Summerfeldt, Kloosterman, Antony, McCabe, \& Parker, 2011), así como los trastornos esquizoide y esquizotípico (Montgomery, McCrimmon, Schwean, \& Saklofske, 2010).

La inteligencia emocional también se ha vinculado a tendencias temperamentales, como los Cinco Grandes factores de personalidad, que han sido relacionados con IE (Siegling, Furnham, $\&$ Petrides, 2015), sobre todo el neuroticismo y la extraversión, seguidos de la responsabilidad y, en menor grado, la cordialidad y la apertura. Igualmente, se han identificado relaciones entre algunas de las escalas de inteligencia emocional y la autoestima (Cabello, Fernández-Berrocal, Ruiz-Aranda, \& Extremera-Pacheco, 2006) lo que fomenta la satisfacción vital y el ajuste psicológico (Reina \& Oliva, 2015), y actúa como un factor protector frente a los problemas de salud mental en adultos jóvenes (Sharaf, Thompson, \& Walsh, 2009).

Un autoconcepto global adecuado en adolescentes predice la implicación conductual y emocional (Rodríguez-Fernández, Ramos-Díaz, Ros, \& Zuazagoitia, 2018), identificándose una vinculación entre IE y autoestima que, a su vez, constituye un potenciador de salud mental en pacientes con ansiedad y/o depresión (Góngora $\&$ Casullo, 2009). Se ha analizado el efecto del rendimiento académico sobre la IE en adolescentes, no dándose diferencias entre cursos consecutivos, pero sí cuando se contrasta entre edades extremas (Quinto-Medrano \& Roig-Vila, 2015); hecho que, según los autores, se puede relacionar con diferencias de autoestima durante el proceso madurativo.

Además, se han hallado diferentes perfiles de inteligencia emocional, en función del peso de cada una de sus tres dimensiones en la manifestación de la IE global, no incidiendo del mismo modo cada una de ellas en el ajuste psicológico de los individuos (Gázquez, Pérez-Fuentes, Díaz-Herrero, García-Fernández, \& Inglés, 2015).

Teniendo en cuenta los hallazgos de investigación, resulta de especial interés conocer las derivaciones del constructo de IE y su relación con variables sociodemográficas, temperamentales y de ajuste psicológico en estudiantes de distintos niveles académicos y de distintas ramas de estudio. Por esta razón, el objetivo general del presente trabajo fue analizar la inteligencia emocional en una muestra de de 147 estudiantes adultos, pertenecientes a distintos niveles académicos y ramas de 
estudios, teniendo como objetivos específicos los siguientes: 1) Analizar la asociación de la IE con variables sociodemográficas, de personalidad y de ajuste psicológico (autoestima, apoyo social y sintomatología general); 2) Conocer el poder explicativo de las variables sociodemográficas y de ajuste psicológico sobre cada una de las escalas de IE; 3) Comparar el nivel de IE de tres submuestras de participantes: estudiantes de formación profesional, de grado y de máster; 4) Analizar las diferencias entre casos extremos en cada escala de IE en características sociodemográficas, autoestima, apoyo social y sintomatología general y 5) Contrastar las puntuaciones de IE, autoestima y apoyo social de estudiantes de Ciencias Sociales y de Ciencias de la Salud con estudiantes de Ingeniería Informática.

Igualmente, se plantearon las siguientes hipótesis:

1. La percepción emocional presentará menos asociaciones significativas y estará explicada en menor medida por el ajuste psicológico, que la comprensión y la regulación emocional.

2. La IE será mayor a medida que los estudiantes pertenezcan a niveles académicos más altos -formación profesional, grado o máster universitario-, debido a la mayor formación académica y al propio proceso de maduración personal.

3. Los participantes con puntuaciones altas en las escalas de IE, informarán de mayor autoestima y apoyo social y menor sintomatología general.

4. Los estudiantes de Ciencias Sociales y Ciencias de la Salud puntuarán más alto en todas las escalas de IE, autoestima y apoyo social que los estudiantes de Ingeniería Informática.

\section{Método}

\section{Participantes}

La muestra consta de 147 estudiantes, 92 mujeres (62.6\%) y 55 hombres (37.4\%). El rango de edad se situó entre 19 y 36 años $(M=24.81 ; D E$ = 3.37). Un $49 \%$ son estudiantes universitarios de grado, el $19 \%$ de Máster, un $15.6 \%$ son estudiantes de Ciclo Formativo, un $15 \%$ prepara Oposiciones y un $1.4 \%$ estudia Doctorado. De todos ellos, casi un $71 \%$, además de estudiar, también trabaja. El 51.7 \% tiene pareja estable, y un $49.7 \%$ continúa viviendo en el domicilio familiar (Tabla 1).

\section{Tabla 1}

Descripción sociodemográfica de la muestra de estudio $(N=147)$

\begin{tabular}{ccc}
\hline Características Sociodemográficas & Frecuencia & $\begin{array}{c}\text { Porcentaje } \\
(\%)\end{array}$ \\
\hline Sexo: & 92 & 62.6 \\
- Mujeres & 55 & 37.4 \\
- Hombre & & \\
Nivel Estudios: & 23 & 15.6 \\
- Formación Profesional/Ciclo Formativo & 72 & 49 \\
- Estudios Universitarios de Grado & 22 & 15 \\
- Preparación Oposiciones & 28 & 19 \\
- Estudios Universitarios de Máster & 2 & 1.4 \\
- Estudios Universitarios de Doctorado & & \\
Pareja estable: & 76 & 51.7 \\
- Sí & 71 & 48.3 \\
- No & 73 & \\
Tipo de residencia: & 34 & 49.7 \\
- Domicilio familiar & 31 & 23.1 \\
- Domicilio conyugal & 8 & 21.1 \\
- Piso compartido & 1 & 5.4 \\
- Vivienda unipersonal & & 0.7 \\
- Residencia universitaria &
\end{tabular}

Para llevar a cabo el último objetivo, se ha considerado a los participantes del presente estudio que pertenecían a estudios de Ciencias Sociales y Ciencias de la Salud (grupo de estudio) y se han contrastado con una muestra de estudiantes de Ingeniería Informática (grupo comparativo). Con el fin de homogeneizar ambas muestras a nivel sociodemográfico, únicamente se han considerado a los hombres, dado que en el grupo comparativo fueron prácticamente la totalidad. Así, la muestra quedó conformada por 112 hombres, 56 en cada grupo, de entre 21 y 28 años; en el grupo de estudio $(M=23.92 ; \mathrm{DE}=$ 1.83), teniendo un $52.7 \%$ una relación de pareja estable, y en el grupo comparativo $(M=23.71$; $D E=1.95)$ y un $46.4 \%$ con pareja estable. 


\section{Instrumentos}

Escala Rasgo de Metaconocimiento Emocional/ The Trait Meta-Mood Scale (TMMS-24) (Salovey, Mayer, Goldman, Turvey, $\mathcal{E}$ Palfai, 1995)

Se utilizó la versión española reducida (Fernández-Berrocal et al., 2004). Esta Esacla mide la IE percibida en población no clínica, permitiendo conocer cómo prestar atención a los sentimientos (percepción emocional), conocer cómo son (comprensión emocional), y tener capacidad para regular el pensamiento positivo a partir de los estados emocionales (regulación emocional). Está formada por 24 ítems, ocho por cada escala, con respuesta tipo Likert de 5 puntos, desde 1: nada de acuerdo hasta 5: totalmente de acuerdo. La consistencia interna (alpha de Cronbach) obtenida fue de 0.92 para percepción emocional, 0.9 para comprensión emocional y 0.84 para regulación emocional.

Inventario de los Cinco Grandes Factores de Personalidad/Big Five Inventory (BFI-10) (Gosling, Rentfrow, Eु Swann, 2003)

Se trata de una escala reducida que evalúa los cinco grandes factores de personalidad a través de 10 ítems, dos para cada una de las cinco dimensiones: extraversión, cordialidad, responsabilidad, neuroticismo y apertura, con una escala de respuesta tipo Likert de 5 puntos, desde 1: muy en desacuerdo a 5: muy de acuerdo. Los índices de consistencia interna fueron: extraversión $(\alpha=0.66)$, neuroticismo $(\alpha=0.65)$, cordialidad $(\alpha=0.07)$, responsabilidad $(\alpha=$ $0.51)$ y apertura $(\alpha=0.57)$.

Escala de Autoestima de Rosenberg/The Rosenberg Self-Esteem Scale (RSE) de Rosenberg (1965), en su versión española (Martín-Albo, Núñez, Navarro, Eु Grijalvo, 2007)

La escala mide la autoestima general, a partir de los sentimientos positivos y negativos sobre el yo, permitiendo conocer el respeto y la autoaceptación. Está formada por 10 ítems: cinco enunciados positivamente y otros cinco, negativamente. Utiliza una escala tipo Likert de cuatro puntos, que van desde 1: muy en desacuerdo a 4: muy en acuerdo. La consistencia de la escala alcanzó un alpha de Cronbach de 0.82.

Cuestionario de Apoyo Social/Duke Functional Social Support Scale (UNC-11) (Broadhead, Gehlbach, Degruy, 8 Kaplan, 1998)

Se empleó la escala adaptada a la población española por Bellón-Saameño, DelgadoSánchez, Luna del Castillo y LardelliClaret (1996). Es un cuestionario breve y multidimensional de 11 ítems que evalúa el grado de comunicación con otros, la empatía y tener cubiertas las necesidades. Cuenta con una escala tipo Likert de cinco puntos, que va desde 1: mucho menos de lo que deseo a 5: tanto como deseo. La consistencia interna alcanzó un $\alpha=0.9$.

Cuestionario de Salud General/General Health Questionnaire (GHQ-28) (Goldberg $\mathcal{E}$ Hillier, 1979)

Se utilizó la versión de 28 ítems traducida al español por Lobo, PérezEcheverría y Artal (1986). Permite detectar la siguiente sintomatología psicopatológica: síntomas somáticos, ansiedad e insomnio, disfunción social y depresión. Utiliza una escala tipo Likert, en la que se asignan valores de 0 a 3 . Los valores alpha de Cronbach fueron: síntomas somáticos $(\alpha=0.67)$, ansiedad-insomnio $(\alpha=$ $0.9)$, disfunción social $(\alpha=0.59)$ y depresión $(\alpha$ $=0.85$ ).

\section{Procedimiento}

La batería de evaluación fue aplicada en línea con una duración aproximada de 15 minutos, a una muestra de estudiantes pertenecientes a distintos niveles académicos. La colaboración fue voluntaria y anónima, dando los participantes su 
consentimiento informado para utilizar los datos únicamente con fines de investigación. Se trata de un diseño transversal, con una muestra de conveniencia, ex post facto. Los datos obtenidos fueron computarizados en una base de datos SPSS, versión 21.

Se realizaron análisis correlacionales entre las escalas de IE y variables sociodemográficas, personalidad (excepto cordialidad dada su baja fiabilidad) autoestima, apoyo social y sintomatología general. Además, se incluyeron tres análisis de regresión múltiple, uno sobre cada escala -variables criterio-, siendo variables predictoras: las sociodemográficas, de personalidad, de autoestima, de apoyo social y de sintomatología general. Se analizaron posibles diferencias a través de un ANOVA en percepción, comprensión y regulación emocional, personalidad, autoestima, apoyo social y las variables de sintomatología general, en función del nivel académico. Además, se estudiaron las diferencias entre casos extremos en cada escala de IE. Teniendo en cuenta el número de participantes en cada grupo, se llevaron a cabo análisis de diferencias de medias con la prueba $t$ para Percepción Emocional y con la U de Mann Whitney para Comprensión y para Regulación Emocional. Finalmente, se realizó contraste de medias (prueba $t$ ), considerando una parte de la muestra (estudiantes pertenecientes a carreras de Ciencias Sociales y Ciencias de la Salud) con los datos de una muestra de estudiantes de Ingeniería Informática.

\section{Resultados}

Los análisis correlacionales entre la IE y variables sociodemográficas, de personalidad y de ajuste psicológico se presentan en la Tabla 2.

\section{Tabla 2}

Análisis correlacionales bivariados ( $r$ de Pearson) de las variables de IE con las sociodemográficas, de personalidad y de ajuste psicológico $(N=147)$

\begin{tabular}{|c|c|c|c|}
\hline $\begin{array}{l}\text { Variables sociodemográficas, de } \\
\text { personalidad y ajuste psicológico }\end{array}$ & $\begin{array}{l}\text { Percepción } \\
\text { Emocional }\end{array}$ & $\begin{array}{l}\text { Comprensión } \\
\text { Emocional }\end{array}$ & $\begin{array}{l}\text { Regulación } \\
\text { Emocional }\end{array}$ \\
\hline Sexo & $-0.18 *$ & -0.02 & 0.1 \\
\hline Edad & 0.07 & $0.2 *$ & $0.18 *$ \\
\hline Tipo de residencia & 0.08 & -0.1 & -0.004 \\
\hline Pareja estable & 0.16 & $0.24 * *$ & 0.12 \\
\hline Nivel Estudios & 0.001 & 0.05 & 0.008 \\
\hline Situación laboral & $0.19 *$ & 0.15 & 0.13 \\
\hline Extraversión & 0.01 & 0.05 & $0.23^{* *}$ \\
\hline Neuroticismo & $0.25^{* *}$ & -0.15 & $-0.22^{* * *}$ \\
\hline Responsabilidad & 0.13 & $0.22 * *$ & $0.33 * * *$ \\
\hline Apertura a la experiencia & 0.09 & $0.34 * * *$ & $0.28 * * *$ \\
\hline Autoestima & $-0.21 * *$ & $0.3 * * *$ & $0.33 * * *$ \\
\hline Apoyo Social & 0.11 & $0.31 * * *$ & $0.28^{* * *}$ \\
\hline Disfunción Social & -0.01 & $0.22 * *$ & $0.22 * *$ \\
\hline Depresión & $0.25^{* *}$ & $-0.22 * *$ & $-0.25^{* * *}$ \\
\hline Síntomas Somáticos & $0.23^{* *}$ & $-0.23 * *$ & -0.13 \\
\hline Ansiedad-Insomnio & $0.24 * *$ & $-0.24 * *$ & -0.13 \\
\hline
\end{tabular}

La percepción emocional mantiene asociaciones con sexo $(r=-0.18)$, situación laboral $(r=0.19)$, neuroticismo $(r=0.25)$, autoestima $(r=-0.21)$, depresión $(r=0.25)$, síntomas somáticos $(r=0.23)$ y ansiedadinsomnio $(r=0.24)$. La comprensión emocional presenta relaciones significativas positivas con edad $(r=0.2)$, pareja estable $(r=0.24)$, apertura a la experiencia $(r=0.34)$, responsabilidad $(r=$ $0.22)$, autoestima $(r=0.3)$, apoyo social $(r=$ $0.31)$ y disfunción social $(r=0.22)$; y negativas con depresión $(r=-0.22)$, síntomas somáticos $(r=-0.23)$ y ansiedad-insomnio $(r=-0.24)$. Por último, la regulación emocional se asocia positivamente con edad $(r=0.18)$, autoestima $(r=0.33)$, responsabilidad $(r=0.33)$, apertura a la experiencia $(r=0.28)$, extraversión $(r=$ $0.23)$, apoyo social $(r=0.28)$ y disfunción social $(r=0.22)$. Las asociaciones negativas se dan con neuroticismo $(r=-0.22)$ y depresión $(r=-0.25)$.

Se llevaron a cabo tres análisis de regresión lineal (Tabla 3) sobre percepción, comprensión y regulación emocional, y como variables predictoras: las sociodemográficas (excepto tipo de residencia y nivel de estudios, sin asociaciones con IE), personalidad, autoestima, apoyo social y sintomatología general. Se controló la multicolinealidad, con niveles de tolerancia adecuados de las variables predictoras. 
Las variables que predijeron la percepción emocional fueron: depresión $(b=0.31)$, apoyo social $(b=0.25)$, neuroticismo $(b=0.23)$ y situación laboral $(\mathrm{b}=0.23)$. Todas ellas explicaron el $18 \%$ de dicha variable $\left[F_{(4,142)}\right.$ $=8.97 ; p=0$ ]. La comprensión emocional fue explicada por: apertura a la experiencia $(\mathrm{b}=$ $0.3)$, tener pareja estable $(b=0.21)$ y síntomas somáticos $(\mathrm{b}=-0.2)$, explicando el $22 \%$ de dicha variable $\left[F_{(4,142)}=11.46 ; p=0\right]$. Las variables que predijeron la regulación emocional fueron: apertura a la experiencia $(b=0.24)$, autoestima $(b=0.2)$, responsabilidad $(b=0.24)$ y extraversión $(b=0.17)$, que explican el $23 \%$ de dicha variable $\left[F_{(4,142)}=11.83 ; p=0\right]$.

\section{Tabla 3}

Análisis de regresión múltiple de variables sociodemográficas y de ajuste psicológico sobre percepción, comprensión y regulación emocional $(\mathrm{N}=147)$

\begin{tabular}{|c|c|c|c|c|c|c|c|}
\hline \multirow{2}{*}{$\begin{array}{l}\text { Variables incluidas } \\
\text { en el modelo }\end{array}$} & \multicolumn{7}{|c|}{ Percepción Emocional } \\
\hline & $\beta$ & $t$ & $p$ & Cor. Par. & $R^{2}$ Cor. & $F$ & $p$ \\
\hline Depresión & 0.31 & 3.68 & 0 & 0.29 & 0.18 & 8.97 & 0 \\
\hline Apoyo social & 0.25 & 3.04 & 0.003 & 0.25 & & & \\
\hline Neuroticismo & 0.23 & 2.88 & 0.005 & 0.23 & & & \\
\hline Situación laboral & 0.23 & 3.07 & 0.003 & 0.25 & & & \\
\hline \multirow{2}{*}{$\begin{array}{l}\text { Variables incluidas } \\
\text { en el modelo }\end{array}$} & \multicolumn{7}{|c|}{ Comprensión Emocional } \\
\hline & $\beta$ & $t$ & $p$ & Cor. Par. & $R^{2}$ Cor. & F & $p$ \\
\hline Apertura & 0.3 & 4.12 & 0 & 0.33 & 0.22 & 11.46 & 0 \\
\hline Pareja estable & 0.21 & 2.69 & 0.008 & 0.22 & & & \\
\hline Síntomas somáticos & -0.2 & -2.56 & 0.011 & -0.21 & & & \\
\hline \multirow{2}{*}{$\begin{array}{l}\text { Variables incluidas } \\
\text { en el modelo }\end{array}$} & \multicolumn{7}{|c|}{ Regulación Emocional } \\
\hline & $\beta$ & $t$ & $p$ & Cor. Par. & $R^{2}$ Cor. & $F$ & $p$ \\
\hline Apertura & 0.24 & 3.22 & 0.002 & 0.26 & 0.23 & 11.83 & 0 \\
\hline Autoestima & 0.2 & 2.49 & 0.014 & 0.2 & & & \\
\hline Responsabilidad & 0.24 & 3.2 & 0.002 & 0.26 & & & \\
\hline Extraversión & 0.17 & 2.23 & 0.027 & 0.18 & & & \\
\hline
\end{tabular}

Se realizó una comparación de la IE entre los tres niveles académicos (Ciclo Formativo, Grado Universitario y Máster Universitario) con el ANOVA de Kruskal-Wallis, dados los tamaños de cada una de las tres submuestras. No se dieron diferencias significativas en ninguno de los contrastes: percepción $\left(\chi^{2}=0.016 ; p=0.992\right)$, comprensión $\left(\chi^{2}=0.549 ; p=0.76\right)$ y regulación emocional $\left(\chi^{2}=0.468 ; p=0.791\right)$.

Además, se analizaron las diferencias (prueba $t$ o U de Mann-Whitney) en variables sociodemográficas y de ajuste psicológico, entre estudiantes con puntuaciones extremas en IE, según el criterio propuesto por Passos-Simancas (2014). Para cada escala, se utilizaron como puntos de corte en función de que se tratara de hombres o de mujeres, percentiles entre 75 y 80 \#grupos altos\# y entre 20 y 25 \#grupos bajos\#. Dichos resultados se presentan en la Tabla 4.

\section{Tabla 4}

Contrastes en variables sociodemográficas y de ajuste psicológico entre grupos extremos en: Percepción Emocional (PE), Comprensión Emocional (CE) y Regulación Emocional (RE)

\begin{tabular}{lcccccc}
\hline \multirow{2}{*}{$\begin{array}{c}\text { Variables de } \\
\text { contraste }\end{array}$} & $\begin{array}{c}\text { Percepción Emoc. } \\
\text { Baja PE }(N=35) \\
\text { Alta PE }(N=31)\end{array}$ & \multicolumn{2}{c}{\begin{tabular}{c} 
Comprensión Emoc. \\
Baja CE $(N=35)$ \\
\cline { 2 - 7 } Alta CE $(N=18)$
\end{tabular}} & $\begin{array}{c}\text { Regulación Emoc. } \\
\text { Baja RE }(N=19) \\
\text { Alta RE }(N=27)\end{array}$ \\
\cline { 2 - 7 } Edad & $t$ & $p$ & $z$ & $p$ & $z$ & $p$ \\
Tipo de residencia & -0.47 & 0.64 & -2.94 & 0.003 & -1.69 & 0.09 \\
Pareja estable & -1.71 & 0.39 & -0.204 & 0.84 & -0.42 & 0.68 \\
Nivel de estudios & 0.63 & 0.53 & -2.97 & 0.003 & -1.04 & 0.3 \\
Situación laboral & -2.62 & 0.01 & -0.85 & 0.54 & -0.75 & 0.46 \\
Autoestima & 1.64 & 0.11 & -2.1 & 0.04 & -2.91 & 0.004 \\
Apoyo Social & -0.57 & 0.57 & -3.43 & 0.001 & -2.97 & 0.003 \\
Síntomas Somáticos & -2.25 & 0.03 & -2.37 & 0.02 & -1.87 & 0.06 \\
Ansiedad-Insomnio & -2.22 & 0.03 & -2.13 & 0.03 & -0.73 & 0.47 \\
Disfunción Social & -0.91 & 0.37 & -1.99 & 0.05 & -2.6 & 0.009 \\
Depresión & -2.34 & 0.02 & -2.47 & 0.014 & -2.29 & 0.022 \\
\hline
\end{tabular}

El grupo de alta percepción emocional es más probable que esté activo a tiempo completo $(t=-2.62 ; p=0.01)$, presenta más síntomas somáticos $(t=-2.25 ; p=0.03)$, ansiedadinsomnio $(t=-2.22 ; p=0.03)$ y depresión $(t$ $=-2.34 ; p=0.02)$. Los participantes altos en comprensión emocional tienen más edad $(z=$ -2.94; $p=0.003)$, pareja estable $(z=-2.97$; $p=0.003)$, presentan más autoestima $(z=$ $-2.1 ; p=0.04)$, apoyo social $(z=-3.43 ; p=$ $0.001)$ y disfunción social $(z=-1.99 ; p=0.046)$; y menos síntomas somáticos $(z=-2.37 ; p=$ $0.018)$, ansiedad e insomnio $(z=-2.13 ; p=$ $0.033)$ y depresión $(z=-2.47 ; p=0.014)$ que los de puntuaciones bajas. Por último, las personas con alta regulación emocional, presentan mayor autoestima $(z=-2.91 ; p=0.004)$, apoyo social $(z$ $=-2.97 ; p=0.003)$, disfunción social $(z=-2.6$; $p=0.009)$ y menos depresión $(z=-2.29 ; p=$ 0.022 ).

Por último, se hizo un contraste considerando una muestra de estudiantes de Ingeniería Informática, que incluye únicamente a los 
hombres, dado que en este grupo comparativo, fueron prácticamente la totalidad. En el grupo de estudio, solamente se tuvo en cuenta a aquellos estudiantes de carreras de Ciencias Sociales y de Ciencias de la Salud. Así, cada una de las submuestras estuvo formada por 56 hombres ( $N$ = 112) (Tabla 5). Los análisis chi cuadrado mostraron que los grupos fueron homogéneos en edad $\left.\chi^{2}(7)=9.58 ; p=0.21\right)$ y en tener pareja $\left.\chi^{2}(1)=1.51 ; p=0.22\right)$.

Los contrastes de medias con la prueba $t$, únicamente mostraron diferencias en apoyo social $(t=15.9 ; p=0 ; d=3.03)$ y en percepción emocional $(t=3.5 ; p=0.001 ; d=0.67)$, siendo las puntuaciones más bajas en el grupo de estudiantes de Ingeniería Informática.

\section{Tabla 5}

Diferencias de medias (prueba t) entre el grupo de estudio $(N=56)$ y el grupo comparativo $(N=56)$

\begin{tabular}{|c|c|c|c|c|c|c|c|}
\hline \multirow{2}{*}{$\begin{array}{c}\text { Variables de Inteligencia } \\
\text { Emocional y Ajuste } \\
\text { Psicológico }\end{array}$} & \multicolumn{2}{|c|}{$\begin{array}{l}\text { G. de estudio } \\
(N=56)\end{array}$} & \multicolumn{2}{|c|}{$\begin{array}{l}\text { G. comparativo } \\
\qquad(N=56)\end{array}$} & \multirow[t]{2}{*}{$t$} & \multirow[t]{2}{*}{$p$} & \multirow{2}{*}{$\begin{array}{c}d \\
\text { Cohen }\end{array}$} \\
\hline & M & $D E$ & M & $D E$ & & & \\
\hline & 29 & & & & .5 & & \\
\hline & 28. & 4 & & & 0.44 & 0. & \\
\hline Reg & 29.2 & 6. & 28.8 & 5.1 & 0.34 & 0.74 & 0.07 \\
\hline Aut & 32.3 & 5. & 31.3 & 5.1 & 0.96 & 0.34 & 0.18 \\
\hline Apoyo Social & 46.5 & 6.1 & 30.4 & 4.4 & 15.9 & 0 & 3.03 \\
\hline
\end{tabular}

\section{Discusión}

Los resultados mostraron que la IE se encuentra modulada por la personalidad, fundamentalmente apertura a la experiencia, hallazgo también obtenido entre universitarios canadienses (Saklofske, Austin, \& Minski, 2003) y peruanos (Hemani, Aquino, \& Araujo, 2017). Características como tener pareja estable, disponer de apoyo social o no manifestar sintomatología somática, también parecen indicar mayor comprensión emocional. La responsabilidad adquiere un mayor protagonismo a medida que se hace más complejo el constructo de IE, quizás porque refleja mayor capacidad empática (Alecsiuk, 2015) y cierta regulación en contextos de interacción interpersonal y de resolución de conflictos (Larsen, 2000). Esto, unido a la extraversión y a la autoestima, puede incidir en una mayor manifestación de dicha regulación emocional, proporcionando un mecanismo adaptativo a los estudiantes para poder enfrentarse eficazmente al estrés de la vida académica (Cabanach, Souto-Gestal, GonzálezDoniz, \& Corrás, 2018).

En la primera hipótesis se plantea que la percepción emocional presentará una menor vinculación con ajuste psicológico que el resto de escalas de IE. Esto no se confirma totalmente, ya que, más que un menor número de relaciones o ausencia de ellas, se da un patrón correlacional inverso al que mantienen comprensión y regulación emocional. A partir de la investigación realizada hasta el momento, que muestra menor vinculación de dicha escala de IE con otras variables psicológicas, el presente estudio halló asociaciones opuestas con respecto al resto de variables. Una posible explicación puede estar basada en que la depresión y el neuroticismo parecen ejercer influencia sobre la percepción emocional, resultado que, aunque no va en la línea de algunos hallazgos (de Alba \& Colorado, 2012), sí parece corroborar otros estudios (ExtremeraPacheco \& Fernández-Berrocal, 2006; Ferrando, Ferrándiz, Prieto, \& Hernández, 2007), que obtienen vinculación positiva de la percepción emocional con estrés (Serrano \& Andreu, 2016) o con depresión como predictor significativo de ideación suicida en estudiantes de Enfermería (Aradilla-Herrero, Tomás-Sábado, \& GómezBenito, 2014). El neuroticismo hace atender en mayor medida y preocuparse en exceso por los estados emocionales (Enríquez-Anchondo, 2011), pudiendo redundar esto en una mayor presencia de sintomatología depresiva (Barraza-López, Muñoz-Navarro, \& BehrensPérez, 2017; Fernández-Berrocal \& ExtremeraPacheco, 2006; Hodzic, Ripoll, Costa, \& Zenasni, 2016).

Estos resultados sobre la percepción emocional pueden ser debidos al contenido de los ítems de esta escala del TMMS-24, excesivamente focalizados en las emociones y su semejanza semántica con cierta sintomatología neurótica y depresiva, tal y como han propuesto Goldenberg, Matheson y Mantler (2006). Dicha tendencia excesiva de la percepción emocional a centrarse 
en las emociones puede resultar desadaptativa cuando no se acompaña de comprensión y regulación emocional (Cabanach et al., 2018), perpetuando el estado de ánimo negativo a través de un afrontamiento desadaptativo (Augusto-Landa \& López-Zafra, 2010). En este sentido, Fernández-Berrocal y ExtremeraPacheco (2006) proponen utilizar variables menos influenciadas por sesgos de los autoinformes, aunque relacionadas con el funcionamiento emocional (Lizeretti et al., 2006; Maizal \& González, 2006).

Aunque podría esperarse que la maduración cognitivo-afectiva de edades superiores y, por tanto, un más alto nivel académico, pudiera influir en la manifestación de mayor IE, no se hallaron diferencias en este sentido, por lo que, teniendo en cuenta este resultado, la segunda hipótesis no se cumpliría. Tampoco Sanmartín, Gonzálvez y Vicent (2018) encontraron tales diferencias, al contrastar la IE en alumnos de cualificación profesional inicial con alumnos de ciclos profesionales superiores. Y los estudios que hacen comparaciones en función de la edad, tampoco han hallado diferencias (Cazalla-Luna \& Molero, 2014; Serrano \& Andreu, 2016).

En cuanto a las diferencias en puntuaciones extremas en IE, aunque se siguen dando asociaciones importantes de percepción emocional con ansiedad y depresión -aspecto ya comentado-, aparecen patrones diferentes con respecto a comprensión y regulación en las que puntuar alto se asocia a mayor ajuste psicológico en forma de alta autoestima y apoyo social y baja depresión, por lo cual, se podría afirmar que la tercera hipótesis se confirma. También Calero, Barreyro y Injoque-Ricle (2018) hallaron relación entre dichas escalas de IE y autoestima. Sin embargo, se observó una vinculación de estas dos escalas de IE con disfunción social, hecho que podría tener que ver con aspectos particulares de estos estudiantes, con una tendencia generalizada hacia la inseguridad a la hora de llevar a cabo las tareas propias del alumnado, especialmente, cuando estas se relacionan con una toma de decisiones adecuada y un modo útil y eficaz de actuación. Es reconocida la relación entre afectos, motivación y rendimiento académico, aunque se desconoce exactamente cómo se produce la vinculación con inteligencia emocional (Vargas \& Montero, 2016).

En el presente trabajo se obtuvieron puntuaciones más altas en percepción emocional y apoyo social entre los estudiantes de Ciencias Sociales y Ciencias de la Salud. La investigación halló que el área de estudio puede vincularse a los enfoques de aprendizaje de estudiantes universitarios. Se encontraron relaciones negativas entre IE y niveles de estrés en alumnado de Ciencias de la Salud (Birks, McKendree, \& Watt, 2009), e incluso, niveles más altos de IE entre estudiantes de carreras de Psicología o Enfermería, frente a alumnado de Ingeniería (Valverde-Portales, 2016). Es posible que en estas ramas de conocimiento con mayor implicación de servicio, el estudiantado disponga de más opciones para entrenar dichas capacidades emocionales dado que deben hacer frente a situaciones con carga emocional intensa que es necesario regular, además de la necesidad de promover relaciones adecuadas con el grupo de trabajo. Mientras, aquellos estudiantes que eligen carreras de tecnología probablemente centren su objetivo en la tarea, no otorgando tanto valor a la manifestación de emociones, por lo que su manejo puede ser más limitado.

Las capacidades emocionales contribuyen a una mayor disponibilidad y apoyo social percibido (Azpiazu, Esnaola, \& Sarasa, 2015). El déficit en percepción emocional hallado entre los estudiantes de Ingeniería puede estar asociado a su menor apoyo social, del que también informan. Este hecho podría ser habitual entre estos estudiantes, quizás más centrados en el manejo tecnológico que en el de las emociones en el contexto social. Aunque debemos mantener en perspectiva los hallazgos sobre la vinculación entre percepción emocional y desajuste psicológico, en forma de neuroticismo y sintomatología psicopatológica. Quizás, estos estudiantes no presenten esta problemática y ello tenga que ver con sus puntuaciones más bajas en percepción emocional. En cualquier caso, deben tomarse con cautela estos resultados, pues no son lo suficientemente contundentes como para interpretarlos en un sentido o en el opuesto. 
Además, otra razón importante de las diferencias entre los estudiantes pertenecientes a estudios de ramas opuestas, puede situarse en sus características temperamentales, o en la posibilidad por verse influidos por el contexto social (Rodríguez-Muñiz, Areces, Suárez-Álvarez, Cueli, \& Muñiz, 2019) que inciden en la elección de carrera, inclinando sus intereses hacia una u otra rama de conocimiento. En este sentido, Peró, Soriano, Capilla, Olmos y Hervás (2015) reportaron que los estudiantes de educación o salud daban más valor a la vocación, mientras que los de ingenierías o arquitectura valoraban en mayor medida las salidas laborales o la utilidad de la propia carrera.

Entre las principales limitaciones del presente estudio destacan: 1) La imposibilidad de establecer relaciones causales entre las variables, dado el carácter correlacional del estudio; 2) El limitado tamaño de las muestras, tanto en el grupo de estudio como en el de contraste; 3) La utilización únicamente del sexo masculino en el contraste entre grupos, dado que el grupo crítico estaba formado básicamente por hombres. Se debe tener presente que suelen darse diferencias de sexo en las escalas de IE (Mayer et al., 2000; Salovey \& Mayer, 1990), puntuando las mujeres más alto en percepción emocional y los hombres en regulación emocional (ExtremeraPacheco et al., 2007; Serrano \& Andreu, 2016); 4) El no haber utilizado medidas de habilidad de IE con tareas de ejecución, que permitan abordar el desempeño más que la percepción subjetiva (Serrano \& Andreu, 2016), evitando los efectos de la deseabilidad social que los instrumentos de autoinforme pueden producir sobre la propia capacidad del manejo de las emociones; 5) La escala TMMS-24, destinada a población no clínica, puede limitar su extrapolación a otro tipo de poblaciones y 6) Los ítems que forman parte de la escala de percepción emocional del TMMS-24 tienen una connotación negativa, lo que puede estar incidiendo en su relación positiva con neuroticismo y sintomatología general, y negativa con autoestima.

Los hallazgos de la presente investigación confieren relevancia a la competencia emocional y ajuste psicológico de los estudiantes, que les permita promover una adecuada gestión de sus emociones, particularmente, en aquellos jóvenes que dirigen sus intereses a profesiones tecnológicas, que requieren de poco contacto con otras personas; aunque también, entre los que dedicándose a carreras de Ciencias de la Salud o de Ciencias Sociales, es imprescindible dicho dominio para con los beneficiarios de sus servicios. Teniendo en cuenta su propio proceso madurativo, resulta de gran interés conocer el funcionamiento de ciertas variables de ajuste psicológico, sin duda, con importantes repercusiones en la vida de dichos individuos, tanto durante su preparación académica como en el desarrollo de su carrera profesional, durante la etapa adulta.

\section{Referencias}

Alecsiuk, B. (2015). Inteligencia emocional y desgaste por empatía en terapeutas. Revista Argentina de Clínica Psicológica, 24(1), 43-56. Recuperado de https://bit.ly/2aW02 $9 \mathrm{e}$

Aradilla-Herrero, A., Tomás-Sábado, J., \& Gómez-Benito, J. (2014). Associations between emotional intelligence, depression and suicide risk in nursing students. Nurse Education Today, 34(4), 520-525. https://do i.org/10.1016/j.nedt.2013.07.001

Augusto-Landa, J. M., \& López-Zafra, E. (2010). The impact of emotional intelligence on nursing: An overview. Psychology, 1, 50-58. https://doi.org/10.4236/psych.2010.11008

Azpiazu, L., Esnaola, I., \& Sarasa, M. (2015). Capacidad predictiva del apoyo social en la inteligencia emocional de adolescentes. European Journal of Education and Psychology, 8, 23-29. Recuperado de ht tps://bit.ly/2KoTSAJ

Bar-On, R. (2000). Emotional and social intelligence: Insights from the emotional quotient inventory. En R. Bar-On \& J. D. A. Parker (Eds.), Handbook of Emotional Intelligence (pp. 363-388). San Francisco: Jossey-Bass. 
Barraza-López, R. J., Muñoz-Navarro, N. A., \& Behrens-Pérez, C. C. (2017). Relación entre inteligencia emocional y depresiónansiedad y estrés en estudiantes de medicina de primer año. Revista Chilena de Neuro-Psiquiatría, 55(1), 18-25. https://doi. org/10.4067/S0717-92272017000100003

Bellón-Saameño, J. A., Delgado-Sánchez, A., Luna-del Castillo, J. D. D., \& LardelliClaret, P. (1996). Validez y fiabilidad del cuestionario de apoyo social funcional Duke-UNC-11. Atención Primaria, 18(4), 153-163. Recuperado de https://bit.ly/2mjk $\mathrm{kxG}$

Birks, Y., McKendree, J., \& Watt, I. (2009). Emotional intelligence and perceived stress in healthcare students: A multiinstitutional, multi-professional survey. BMC Medical Education, 9(1), 61. h ttps://doi.org/10.1186/1472-6920-9-61

Boyatziz, R., Goleman, D., \& Rhee, K. (2000). Clustering competence in emotional intelligence: Insights from the emotional competence inventory (ECI). En R. BarOn \& J. D. A. Parker (Eds.), Handbook of emotional intelligence(pp. 343-362). San Francisco: Jossey-Bass.

Broadhead, W. E., Gehlbach, S. H., Degruy, F. V., \& Kaplan, B. H. (1998). The Duke-UNK functional social support questionnaire: Measurement of social support in family medicine patients. Medical Care, 26(7), 709-723. Recuperado de https://bit.ly/2rRv $\mathrm{pZm}$

Cabanach, R. G., Souto-Gestal, A., González Doniz, L., \& Corrás, T. (2018). Afrontamiento y regulación emocional en estudiantes de fisioterapia. UniversitasPsychologica,17(2), 1-13. https:// doi.org/10.11144/Javeriana.upsy17-2.aree

Cabello, R., Fernández-Berrocal, P., Ruiz Aranda, D., \& Extremera-Pacheco, N. (2006). Una aproximación a la integración de diferentes medidas de regulación emocional. AnsiedadyEstrés, 12(2-3), 155-166. Recuperado de https://bit.ly/2LWTyue

Calero, A. D., Barreyro, J. P., \& Injoque-Ricle, I. (2018). Emotional intelligence and self- perception in adolescents. Europe's Journal of Psychology, 14(3), 632-643. https://doi.or g/10.5964/ejop.v14i3.1506

Cazalla-Luna, N., \& Molero, D. (2014). Inteligencia emocional percibida, ansiedad y afectos en estudiantes universitarios. Revista Española de Orientación y Psicopedagogía, 25(3), 56-73. Recuperado de https://www.redalyc.org/pdf/3382/33823 3061007.pdf

de Alba, U. R., \& Colorado, Y. S. (2012). Relación entre inteligencia emocional, depresión y rendimiento académico en estudiantes de Psicología. Psicogente, 15(28), 348-359. Recuperado de https://bit $.1 \mathrm{l} / 2 \mathrm{wPyVcJ}$

Egido, M. P. (2018). La psicologización de la educación: implicaciones pedagógicas de la inteligencia emocional y la psicología positiva. Educación XX1, 21 (1), 303-320. h ttps://doi.org/10.5944/educXX1.16058

Enríquez-Anchondo, H. A. (2011). La inteligencia emocional plena, hacia un programa de regulación emocional basado en la conciencia plena (Tesis doctoral inédita). Universidad de Málaga, Málaga, España. Recuperado de https://bit.ly/2rQ5Etf

Extremera-Pacheco, N., Durán, A., \& Rey, L. (2007). Inteligencia emocional y su relación con los niveles de burnout,engagementy estrés en estudiantes universitarios. RevistadeEducación,342, 239-256. Recuperado de https://bit.ly/2oIm DLK

Extremera-Pacheco, N., \& Fernández-Berrocal, P. (2003). La inteligencia emocional en el contexto educativo: hallazgos científicos de sus efectos en el aula. Revista de educación, 332, 97-116. Recuperado de https://bit.ly/2 AFgOEC

Extremera-Pacheco, N., \& Fernández-Berrocal, P. (2006). Emotional intelligence as predictor of mental, social and physical health in university students. The Spanish Journal of Psychology, 9(1), 45-51. https://do i.org/10.1017/S1138741600005965

Fernández-Berrocal, P., \& Extremera-Pacheco, N. (2006). Special issue on emotional 
intelligence: An overview. Psicothema, 18(Supl.), 1-6. Recuperado de https://bit.ly $12 \mathrm{KwGx} 5 \mathrm{~B}$

Fernández-Berrocal, P., Extremera-Pacheco, N., \& Ramos, N. (2004). Validity and reliability of the Spanish modified version of the Trait Meta-Mood Scale. Psychological Reports, 94(3), 751-755. https://doi.org/10.2466/pr0 $.94 .3 .751-755$

Ferrando, M., Ferrándiz, C., Prieto, M. D., \& Hernández, D. (septiembre, 2007). Exploración de las relaciones entre la inteligencia emocional autopercibida y los rasgos de la personalidad. En P. FernándezBerrocal (Ed.), Libro de resúmenes del I Congreso Internacional de Inteligencia Emocional (p. 84). Málaga: Gráfiko.

Gázquez, J. J., Pérez-Fuentes, M. C., DíazHerrero, Á., García-Fernández, J. M., \& Inglés, C. J. (2015). Perfiles de inteligencia emocional y conducta social en adolescentes españoles. Psicología Conductual, 23(1), 141-160. Recuperado de https://rua.ua.es/dspace/bitstream/1004 5/57933/1/2015_Gazquez_etal_Behavioral Psychology.pdf

Goldberg, D. P., \& Hillier, V. F. (1979). A scaled version of the General Health Questionnaire. Psychological Medicine, 9(1), 139-145. https://doi.org/10.1017/S0033291 700021644

Goldenberg, I., Matheson, K., \& Mantler, J. (2006). The assessment of emotional intelligence: A comparison of performancebased and self-report methodologies. Journal of Personality Assessment, 86(1), 33-45. https://doi.org/10.1207/s15327752j pa8601_05

Goleman, D. (1995). Inteligencia emocional. Barcelona: Kairós.

Goleman, D., \& Davidson, R. J. (2017). Los beneficios de la meditación: la ciencia demuestra cómo la meditación cambia la mente. Barcelona: Editorial Kairós.

Góngora, V. C., \& Casullo, M. M. (2009). Factores protectores de la salud mental: Un estudio comparativo sobre valores, autoestima e inteligencia emocional en población clínica y población general. Interdisciplinaria, 26(2), 183-205. Recuperado de https://bit.ly/2wWOHE6

Gosling, S. D., Rentfrow, P. J., \& Swann, W. B. (2003). A very brief measure of the Big-Five personality domains. Journal of Research in personality, 37(6), 504-528. https://doi.org/ 10.1.1.113.6704\&rep $=$ rep $1 \&$ type $=p d f$

Hemani, L., Aquino, A., \& Araujo, E. (2017). Relación entre el bienestar autopercibido, autoestima, inteligencia emocional, personalidad y razonamiento abstracto en un grupo de estudiantes universitarios. Revista Peruana de Psicologìa y Trabajo Social, 2(1), 49-62. Recuperado de https://bit.ly/2Ir1m1P

Hodzic, S., Ripoll, P., Costa, H., \& Zenasni, F. (2016). Are emotionally intelligent students more resilient to stress? The moderating effect of emotional attention, clarity and repair. Behavioral Psychology/ Psicología Conductual, 24(2), 253-272. Recuperado de https://bit.ly/2YxRwCA

Larsen, R. J. (2000). Toward a science of mood regulation. Psychological Inquiry, 11, 129-141. https://doi.org/10.1207/S1532796 5PLI1103_01

Lenaghan, J. A., Buda, R., \& Eisner, A. B. (2007). An examination of the role of emotional intelligence in work and family conflict. Journal of Managerial Issues, 19(1), 76-94. Recuperado de https://bit.ly/2KyVkNg

Lizeretti, N. P., Oberst, U. E., Chamarro, A., \& Farriols, N. (2006). Evaluation of emotional intelligence in patients with psychopathology: Preliminary results using the TMMS-24 and the MSCEIT. Ansiedad y Estrés, 12(2/3), 355. Recuperado de https ://bit.ly/2rNTu3Y

Lobo, A., Pérez-Echeverría, M. J., \& Artal, J. (1986). Validity of the scaled version of the General Health Questionnaire (GHQ-28) in a Spanish population. Psychological Medicine, 16(1), 135-140. https://doi.org/10 $.1017 /$ S0033291700002579

Maizal, J. B., \& González, A. F. (2006). La inteligencia emocional como predictora de la adaptación psicosocial en el ámbito 
educativo. Resultados de una investigación empírica con estudiantes de la comunidad de Madrid. Ansiedad y Estrés, 12(2-3), 427-439. Recuperado de https://bit.ly/2rPb OnW

Martín-Albo, J., Núñez, J. L., Navarro, J. G., \& Grijalvo, F. (2007). The Rosenberg SelfEsteem Scale: Translation and validation in university students. Spanish Journal of Psychology, 10, 458-467. https://doi.org/10. 1017/S1138741600006727

Martins, A., Ramalho, N., \& Morin, E. (2010). A comprehensive meta-analysis of the relationship between emotional intelligence and health. Personality and Individual Differences, 49(6), 554-564. https ://doi.org/10.1016/j.paid.2010.05.029

Mayer, J. D., Caruso, D., \& Salovey, P. (1999). Emotional intelligence meets traditional standards for an intelligence. Intelligence, 27(4), 267-298. https://doi.org/10.1016/S0 16-2896(99)00016-1

Mayer, J. D., \& Salovey, P. (1993). The intelligence of emotional intelligence. Intelligence, 17(4), 433-442. Recuperado de https://bit.ly/2r4KFV9

Mayer, J. D., Salovey, P., \& Caruso, D. (2000). Emotional intelligence as zeitgeist, as personality, and as a mental ability. En R. Bar-On \& J.D.A. Parker (Eds.), The handbook of emotional intelligence: Theory, development, assessment, and application at home, school, and in the workplace (pp. 92-116). San Francisco: Jossey-Bass.

Mikolajczak, M., Luminet, O., \& Menil, C. (2006). Predicting resistance to stress: Incremental validity of trait emotional intelligence over alexithymia and optimism. Psicothema, 18(Supl.), 79-88. Recuperado de https://bit.ly/2rOyOn5

Molero-Moreno, C., Saiz-Vicente, E., \& EstebanMartínez, C. (1998). Revisión histórica del concepto de inteligencia: una aproximación a la inteligencia emocional. Revista Latinoamericana de Psicología, 30(1), 11-33. Recuperado de https://bit.ly/2FaSmQU

Montgomery, J. M., McCrimmon, A. W., Schwean, V. L., \& Saklofske, D. H.
(2010). Emotional intelligence in Asperger syndrome: Implications of dissonance between intellect and affect. Education and Training in Autism and Developmental Disabilities, 45 (4), 566-582. Recuperado de https://bit.ly/2Io21oB

Passos-Simancas, E. (2014). Características emocionales, familiares y sociales presentes en los niños, niñas y adolescentes en riesgo de explotación laboral. Revista Tendencias y Retos, 19, 45-61. Recuperado de https://bit .ly/2MLzSXk

Peró, M., Soriano, P. P., Capilla, R., Olmos, J. G., $\&$ Hervás, A. (2015). Questionnaire for the assessment of factors related to university degree choice in Spanish public system: A psychometric study. Computers in Human Behavior, 47, 128-138. https://doi.org/10.10 16/j.chb.2014.09.003

Quinto-Medrano, P., \& Roig-Vila, R. (2015). Estudio de la inteligencia emocional en alumnos de enseñanza secundaria: influencia del sexo y del nivel educativo de los estudiantes y su relación con el rendimiento académico. International Studies on Law and Education, 21, 27-38. Recuperado de https://bit.ly/2IqteDm

Reina, M. C., \& Oliva, A. (2015). De la competencia emocional a la autoestima y satisfacción vital en adolescentes. Behavioral Psychology/Psicología Conductual, 23(2), 345-359. Recuperado de https://bit.l $\mathrm{y} / 2 \mathrm{YcgWWh}$

Rodríguez-Fernández, A., Ramos-Díaz, E., Ros, I., \& Zuazagoitia, A. (2018). Implicación escolar de estudiantes de secundaria: la influencia de la resiliencia, el autoconcepto y el apoyo social percibido. Educación XX1, 21 (1), 87-108. https://doi.org/10.5944/edu cXX1.16026

Rodríguez-Muñiz, L. J., Areces, D., SuárezÁlvarez, J., Cueli, M., \& Muñiz, J. (2019). ¿Qué motivos tienen los estudiantes de Bachillerato para elegir una carrera universitaria? Revista de Psicología y Educación, 14(1), 1-15. https://doi.org/10.2 3923/rpye2019.01.167 
Rosenberg, M. (1965). Rosenberg Self-Esteem Scale (RSE). Acceptance and Commitment Therapy. Measures Package, 61, 52. Recuperado de https://bit.ly/2k7jkvU

Saklofske, D. H., Austin, E. J., \& Minski, P. S. (2003). Factor structure and validity of a trait emotional intelligence measure. Personality and Individual Differences, 34(4), 707-721. https://doi.org/10.1016/S0191-88 69(02)00056-9

Salguero, J. M., Fernández-Berrocal, P., RuizAranda, D., Castillo, R., \& Palomera, R. (2011). Inteligencia emocional y ajuste psicosocial en la adolescencia: el papel de la percepción emocional. European Journal of Education and Psychology, 4(2), 143-152. Recuperado de https://bit.ly/2ty4aEW

Salovey, P., \& Mayer, J. D. (1990). Emotional intelligence. Imagination, Cognition and Personality, 9(3), 185-211. https://doi.org/1 0.2190/DUGG-P24E-52WK-6CDG

Salovey, P., \& Mayer, J. D. (1997). What is emotional intelligence? En P. Salovey \& D. J. Sluyter (Eds.), Emotional development and emotional intelligence. Educational implications (pp. 3-31). Nueva York: Basic Books.

Salovey, P., Mayer, J. D., Goldman, S. L., Turvey, C., \& Palfai, T. P. (1995). Emotional attention, clarity and repair: Exploring emotional intelligence using the Trait Meta-Mood Scale. En J. W. Pennebaker (Ed.), Emotion, disclosure, and health (pp. 125-154). Portland, ME: Amer Psychological Assn.

Sanmartín, R., Gonzálvez, C., \& Vicent, M. (2018). Inteligencia emocional en alumnado de formación profesional. Diferencias en función del curso, del género y de la edad. Educar, 54(1), 229-245. https: //doi.org/10.5565/rev/educar.797

Schutte, N. S., Malouff, J. M., Thorsteinsson, E. B., Bhullar, N., \& Rooke, S. E. (2007). A meta-analytic investigation of the relationship between emotional intelligence and health. Personality and Individual Differences, 42 (6), 921-933. https ://doi.org/10.1016/j.paid.2006.09.003
Serrano, C., \& Andreu, Y. (2016). Inteligencia emocional percibida, bienestar subjetivo, estrés percibido, engagement y rendimiento académico de adolescentes. Revista de Psicodidáctica, 21, 357-374. https://doi.org/ 10.1387/RevPsicodidact.14887

Sharaf, A. Y., Thompson, E. A., \& Walsh, E., (2009). Protective effects of self-esteem and family support on suicide risk behaviors among at-risk adolescents. Journal of Child and Adolescent Psychiatric Nursing, 22(3), 160-168. https://doi.org/10.1111/j.1744 $-6171.2009 .00194 . x$

Siegling, A. B., Furnham, A., \& Petrides, K. V. (2015). Trait emotional intelligence and personality: Gender-invariant linkages across different measures of the Big Five. Journal of Psychoeducational Assessment, 33(1), 57-67. https://doi.org/10.1177/0734 282914550385

Summerfeldt, L. J., Kloosterman, P. H., Antony, M. M., McCabe, R. E., \& Parker, J. D. (2011). Emotional intelligence in social phobia and other anxiety disorders. Journal of Psychopathology and Behavioral Assessment, 33(1), 69-78. https://doi.org/10 $.1007 / \mathrm{s} 10862-010-9199-0$

Valverde-Portales, G. (2016). Inteligencia emocional en estudiantes del tercero a quinto año de una universidad privada de Lima Metropolitana y una universidad nacional de la región Pasco (Tesis inédita de maestría). Universidad Peruana, Unión. Recuperado de https://bit.ly/2IK4ZU5

Vargas, M. M., \& Montero, E. (2016). Factores que determinan el rendimiento académico en Matemáticas en la Universidad Nacional de Ingeniería (UNI), Nicaragua: un modelo de ecuaciones estructurales. Universitas Psychologica, 15(4), 1-11. https://doi.org/10 $.11144 / J a v e r i a n a . u p s y 15-4 . f d r a$

\section{Notas}

* Artículo de investigación. 